\title{
Das schwere akute Atemwegssyndrom
}

\author{
P. Tuschy \\ T. Welte \\ R. Marre \\ N. Suttorp \\ J. Lorenz \\ G. Fätkenheuer \\ S. Ewig
}

\section{SARS - Severe Acute Respiratory Syndrome}

Zwischen November 2002 und Februar 2003 wurde in der Provinz Guangdong in Südchina über eine Häufung atypisch verlaufender Pneumonien berichtet. Wegen des vereinzelten Nachweises von Chlamydia pneumoniae gingen die chinesischen Behörden zunächst von einer lokalen Chlamydienepidemie aus. Seit Mitte März 2003 traten auch gehäuft Pneumonien in Hongkong, Vietnam, Taiwan und Singapur auf. Parallel wurde über Erkrankungsfälle in Kanada, Deutschland, Irland, Frankreich, Italien, Rumänien, England und den USA berichtet. Nach eingehenden Recherchen der WHO geht man inzwischen davon aus, dass es sich bei allen gemeldeten Fällen um ein und dieselbe Krankheit handelt, nämlich das schwere akute Atemwegssyndrom unklarer Ätiologie (SARS). Entstanden in Südchina, wurde die Krankheit zunächst nach Hongkong verschleppt. Von hier lässt sich der Ausbreitungsweg und die Ausbreitungsgeschwindigkeit sehr genau verfolgen. Indexpatienten und Cluster konnten in den betroffenen Ländern exakt definiert werden, so dass neben der Suche nach dem Erreger und der Behandlung von Komplikationen vor allem hygienische Maßnahmen und Quarantäne zum Schutz der Bevölkerungen vor der weiteren Ausbreitung im Vordergrund standen. Ende April zählte die WHO mehr als 5050 Krankheitsfälle [1], über 300 infizierte Personen waren zu diesem Zeitpunkt bereits verstorben. Betroffen waren 26 Länder auf sechs verschiedenen Kontinenten [2]. Hierzu muss allerdings bemerkt werden, dass in der überwiegenden Anzahl der betroffenen Länder lediglich importierte Fälle auftraten und eine direkte Ansteckung hier nicht erfolgte. Andererseits sind die Regionen, welche besonders betroffen sind, gerade durch eine hohe Anzahl Patienten gekennzeichnet, die sich durch direkten Kontakt angesteckt haben. Zu diesen Regionen gehören Hongkong (SAR China) und die Provinzen Guangdong, Innere Mongolei, Peking und Shangxi (China), Singapur, Taiwan und Toronto (Kanada) [3].

\section{Charakteristika von SARS}

Als wesentliche Merkmale von SARS lassen sich aus der Entstehungsgeschichte und den Fallberichten einige Punkte ableiten:

1. Der Erreger ist ein bislang unbekanntes Virus aus der Familie der Corona-Viren. Eine ganze Reihe von Fragen zur Epidemiologie und Pathogenese der Erkrankung muss aber zunächst beantwortet werden, um zur Entwicklung eines diagnostischen Testes, wirksamer Medikamente und eines Impfstoffes zu gelangen.

2. Der Erreger verfügt über eine sehr hohe Kontagiosität.

3. Die Übertragung erfolgt durch Tröpfcheninfektion, eine Kontakt- oder Schmierinfektion erscheint zumindest möglich. Aus diesem Grund gelten Angehörige von Patienten, die in sehr engem Kontakt zu den erkrankten Familienmitgliedern leben, und medizinisches Personal sowie deren Angehörige als Risikogruppen (in einigen Regionen sind der überwiegende Anteil der Erkrankten Angehörige von Patienten sowie deren pflegendes und behandelndes medizinisches Personal). Allerdings wurde Ende März über eine Häufung von Erkrankungen in einem bestimmten Wohnblock in einem Viertel Hongkongs berichtet. Es wird vermutet, dass der Erreger sich möglicherweise auch über andere Wege verbreiten kann (Klimaanlage, Wasserversorgung, Müllschluckersystem o. ä.), obwohl dafür bis jetzt jeglicher Beweis fehlt.

4. Die Inkubationszeit beträgt im Mittel 4-7 Tage (Spannbreite $2-10)$.

5. Die Letalität liegt zwischen 5 und $10 \%$. Sie ist somit vergleichbar mit der Letalität bei anderen Pneumonieformen. Allein die in diesem Jahr sehr heftige Influenzaepidemie in Deutschland hat ein vielfaches an Todesopfern gefordert, ohne dass darüber in der Presse ausführlich berichtet wurde. Ob SARS des Weiteren überdurchschnittlich häufig zu schweren Verläufen

Institutsangaben

„Kompetenznetz für Ambulant Erworbene Pneumonie CAPNetz e.V.“, Albert-Einstein-Allee 47, 89069 Ulm Geschäftsstelle CAPNetz e.V., Albert-Einstein-Allee 47, 89069 Ulm, E-mail: peter-tuschy@capnetz.de

Korrespondenzadresse

P. Tuschy · Kompetenznetz für Ambulant erworbene Pneumonie · CAPNetz e.V., Geschäftsstelle . Albert-Einstein-Allee $47 \cdot 89069$ Ulm·E-mail: peter.tuschy@capnetz.de 
führt, oder ob bestimmte prädisponierende Faktoren oder genetische Einflüsse hier für den Verlauf und die Prognose wichtig sind, kann zum gegenwärtigen Zeitpunkt noch nicht beantwortet werden.

\section{Klinik}

Die Weltgesundheitsorganisation hat eine Falldefinition [4] herausgegeben, nach der Verdachtsfälle und mögliche Fälle unterschieden werden. Ein Verdachtsfall liegt vor, wenn ein Patient nach dem 1. Februar 2003 mit Fieber über $38,0^{\circ} \mathrm{C}$ erkrankt, zusätzlich Husten, Luftnot oder Kurzatmigkeit beklagt und innerhalb von 10 Tagen vor Symptombeginn Kontakt zu einem SARSPatienten hatte oder sich in einem der Risikogebiete (Hongkong [SAR China], Provinzen Guangdong, Innere Mongolei, Peking und Shangxi [China], Singapur, Taiwan und Toronto [Kanada]) aufgehalten hat. Ein möglicher Fall liegt vor bei Erfüllen der Kriterien des Verdachtsfalles und zusätzlichem radiologischen Pneumonienachweis oder dem Vorliegen einer ungeklärten Atemwegserkrankung mit Todesfolge sowie einem Autopsiebefund mit Hinweisen auf ein Adult Respiratory Distress Syndrome (ARDS) ohne feststellbare Ursache.

Die Krankheit beginnt nahezu immer mit einer Erhöhung der Körpertemperatur über $38,0^{\circ} \mathrm{C}$ und Husten. Weitere häufige Symptome können Kopfschmerzen, Muskelsteifigkeit, Appetitverlust, Übelkeit, Verwirrtheit, Ausschlag oder Durchfall sein [5].

Um also Patienten mit grippalen Infekten oder „einheimischen“ Infektionen der oberen und unteren Atemwege bzw. Pneumonien von möglichen SARS-Patienten zu unterscheiden, hilft nur die genaue Reise- und Expositionsanamnese weiter.

\section{Diagnostik}

Seit Ende März 2003 verfügt das Bernhard-Nocht-Institut (Tropeninstitut der Universität Hamburg) über einen automatisierten Test zum Nachweis von Coronaviren. Die Anwendung des Testes ist im Einzelfall jedoch kritisch zu hinterfragen. Typischerweise werden Viren erst beim Auftreten von Symptomen ausgeschieden. Auch SARS bildet hier keine Ausnahme. Die Anwendung des Testes bietet deshalb bei symptomlosen Personen mit positiver Reise- oder Expositionsanamnese keine diagnostische Sicherheit und muss Verdachtsfällen und möglichen Fällen vorbehalten bleiben. Infrage kommende Untersuchungsmaterialien sind respiratorische Sekrete, Rachenabstriche und Stuhl.

Weitere diagnostische Empfehlungen des Robert Koch-Institutes zielen auf den Nachweis typischer und atypischer bakterieller und viraler Pneumonieerreger. Die übliche Routinediagnostik bei Verdachts- und möglichen Fällen bleibt davon unbenommen. In jedem Fall entscheidet der behandelnde Arzt über alle durchzuführenden Maßnahmen.

\section{Verlauf}

Initiale Symptome sind Fieber, Husten, Luftnot und Muskelsteifigkeit. Im Blut lassen sich häufig eine Lymphopenie und milde Thrombozytopenie feststellen. Viele Patienten entwickeln sehr schnell im Verlauf ihrer Erkrankung eine radiologisch nachweisbare Pneumonie. Erhöhte Werte für das Enzym Laktat-Dehydrogenase kommen vor. Alle diese Veränderungen sind nicht spezifisch für SARS und können - in Form und Ausprägung unterschiedlich - auch bei anderen Infektionserkrankungen des Respirationstraktes vorkommen. Engmaschige klinische, laborchemische und apparative Untersuchungen haben den Zweck, frühzeitig sich entwickelnde Komplikationen zu erkennen und zu behandeln. Hierzu gehören das ARDS, ein Multi-Organ-Dyfunktions-Syndrom bis hin zum Multiorganversagen oder septische Komplikationen.

\section{Therapie}

Trotz eindeutig identifiziertem Erreger steht bislang keine kausale Therapie zur Verfügung. Aufgrund der Tatsache, dass Coronaviren behüllte RNA-Viren sind, hat man in Einzelfällen Therapieversuche mit Oseltamivir oder Ribavirin und Steroiden unternommen [6,7]. Über die Wirksamkeit dieser Therapieversuche an z.T. kritisch kranken Patienten kann aber keine allgemein gültige Aussage gemacht werden. Eine Behandlungsempfehlung auszusprechen wäre sicherlich falsch, da keine kontrollierte Studie hierzu vorliegt. Außerdem wurde die überwiegende Anzahl der Patienten, die mit antiviralen Substanzen behandelt wurden, zeitgleich mit einer antibiotischen Kombinationstherapie behandelt, so dass eine näherungsweise sichere Aussage in Bezug auf die Wirksamkeit der einzelnen Substanzen unmöglich ist [6].

Eindeutig positiv aus der klinischen Erfahrung haben sich flankierende und unterstützende Maßnahmen erwiesen. Hierzu gehören eine adäquate Flüssigkeitssubstitution, die Sicherung und Unterstützung der Kreislauffunktion, der Sauerstoffversorgung, der Ernährung und der Ausscheidung. Auch hierbei gilt: Die Entscheidung über die Therapie muss vom behandelnden Arzt nach Abwägung aller zur Verfügung stehenden Informationen individuell getroffen werden.

\section{Schutzmaßnahmen}

Dem Thema Schutz kommt bei SARS eine besondere Bedeutung zu. Der überwiegende Teil der Patienten hat sich in sehr engem Kontakt zu SARS-Patienten befunden und sich somit höchstwahrscheinlich infiziert. Damit sind bestimmte Personengruppen einem höheren Risiko ausgesetzt. Dazu zählen direkte Angehörige sowie in medizinischen Bereichen Tätige und deren Angehörige. Alle Empfehlungen zum persönlichen Schutz dieser Personengruppen betreffen umfassende Hygienerichtlinien und Quarantänemaßnahmen unterschiedlicher Strenge. So ist es nach derzeitigem Stand des Wissens ausreichend, eine Person ohne Krankheitssymptome, aber mit entsprechender Reiseanamnese nach Meldung bei den örtlichen Gesundheitsbehörden für 7-10 Tage unter häusliche Quarantäne zu stellen. Ein Verdachtsfall sollte stationär isoliert werden und ein möglicher 
Fall in einem entsprechenden Zentrum behandelt werden. Zum Schutz des Personals sollten die Richtlinien des sog. „barrier nursing" strikt eingehalten werden, wozu Schutzkittel, Einmalhandschuhe, eine eng anliegende Mund-Nasen-Maske (Schutzstufe FFP3) und gfs. eine Schutzbrille gehören. Besonderer Wert muss auf die Händedesinfektion und die Desinfektion tendenziell kontaminierter Flächen gelegt werden. Auch hinsichtlich der baulichen Voraussetzungen gibt es bestimmte Vorkehrungen, die bei der Behandlung eines SARS-Falles zu treffen sind (z. B. Klimaanlagen abstellen, Schleusen usw.) [8].

\section{Fazit}

SARS ist eine bislang unbekannte Infektionskrankheit, die sich durch den internationalen Flugverkehr weltweit ausbreitet. Die bislang gewonnenen Erkenntnisse und empfohlenen Schutzmaßnahmen lassen das Gefährdungspotenzial für Deutschland allerdings als gering erscheinen. Betroffene Personen sollten entsprechend ihrer Symptomatik isoliert werden. Risikogruppen können sich durch adäquate Präventionsmaßnahmen mit hoher Sicherheit vor einer Ansteckung schützen. Ein diagnostischer Test steht zur Verfügung, eine kausale Therapie und eine Impfung stehen derzeit nicht zur Verfügung. Die WHO empfiehlt nicht zwingend notwendige Reisen in Endemiegebiete zu verschieben. Weitere Anstrengungen zur Erforschung der Entstehung, Epidemiologie, Pathophysiologie, Diagnostik und Therapie sind erforderlich und werden weltweit unternommen.

\section{Informationen zu SARS im Internet}

\section{Weltgesundheitsorganisation WHO: www.who.int/csr/sars/en}

Robert Koch-Institut Berlin: www.rki.de

US Centers for Disease Control and Prevention CDC: www.cdc.gov/ncidod/sars

Bernhard-Nocht-Institut Hamburg: www.gesundes-reisen.de/ index $2 . \mathrm{html}$

Weitere aktuelle und interessante Informationen gibt es auf den SARS-Informationsseiten des „Kompetenznetzwerkes für Ambulant Erworbene Pneumonie CAPNetz" unter www.capnetz.de

Das „Department of Diagnostic Radiology and Organ Imaging“ der Chinesischen Universität Hongkong bietet eine Reihe von Röntgenbildern zum Anschauen unter:

http://www.droid.cuhk.edu.hk/

Das „Department of Anaesthesia and Intensive Care“ der Chinesischen Universität Hongkong bietet Informationen und persönliche Erfahrungen zur Behandlung von SARS-Patienten unter: http://www.aic.cuhk.edu.hk/web8/sudden_acute_respiratory_syndrom.htm
Literatur

${ }^{1}$ WHO Genf. Situation Updates - Severe Acute Respiratory Syndrome SARS. http://www.who.int/csr/sarsarchive/en/

2 WHO Genf. Cumulative Number of Reported Cases (SARS). http://www.who.int/csr/sarscountry/en/

${ }^{3}$ WHO Genf. Affected Areas - Severe Acute Respiratory Syndrome SARS. http://www.who.int/csr/sarsareas/en/

${ }^{4}$ WHO Genf. Case Definitions for Surveillance of Severe Acute Respiratory Syndrome (SARS). http://www.who.int/csr/sars/casedefinition/ en/

${ }^{5}$ RKI Berlin. Falldefinition für Schweres Akutes Respiratorisches Syndrom (SARS). http://www.rki.de/INFEKT/AOLG-FALLDEF-ARSUU.PDF

${ }^{6}$ Poutanen SM et al. Identification of Severe Acute Respiratory Syndrome in Canada. http://content.nejm.org/cgi/content/abstract/ NEJMoa030634v1

${ }^{7}$ Joynt G, Gomersall C. SARS - ICU-Topics of the Chinese University of Hong Kong. http://www.aic.cuhk.edu.hk/web8/sudden_acute_respiratory_syndrome.htm

${ }^{8}$ RKI Berlin. Empfehlungen des Robert Koch-Institutes für die Hygienemaßnahmen und Infektionskontrolle bei Patienten mit Schwerem Akutem Respiratorischem Syndrom (SARS). http://www.rki.de/GESUND/HYGIENE/SARS.PDF 